\title{
Pioneering remote-access use of Diamond beamline I19
}

\author{
$\underline{\text { William Clegg }}^{1}$, Natalie T Johnson ${ }^{1}$, Michael R Probert ${ }^{1}$, Paul G Waddell ${ }^{1}$ \\ ${ }^{1}$ School Of Chemistry, Newcastle University, Newcastle Upon Tyne, United Kingdom \\ E-mail: bill.clegg@ncl.ac.uk
}

The small-molecule single-crystal diffraction beamline I19 at the UK's Diamond Light Source has been in operation since 2008, with a regional team of Newcastle and Durham crystallographers among its leading users from the beginning [1]. In 2016 a major upgrade was made to the beamline, introducing a custom-designed diffractometer and a Dectris Pilatus $2 \mathrm{M}$ detector under control of Diamond in-house software. Together with a redesigned sample-handling robot capable of cryogenic operation (as illustrated), this upgrade provided a considerable improvement in data collection speed and capacity. As part of the new developments, we have been pioneering the use of cryogenically mounted samples shipped from the home laboratory in suitable dry dewars, in partnership with the beamline scientists and Diamond software engineers. Recently we were able to conduct part of an experiment by remote control of the robot, diffractometer, and data-processing software from Newcastle, $500 \mathrm{~km}$ away from the facility. This should soon become a routine procedure available to all users of the beamline, at a substantial saving of time and cost with travel to Diamond no longer required. Although such remote access to synchrotron diffraction facilities has been widely used by macromolecular crystallographers for some years, there are additional challenges in adapting the procedure for samples from chemistry and materials science, which display a wider range of behaviour under cryogenic treatment. The limited experience so far has been almost entirely positive and promises well for future development and application.

[1] Nowell, H., Barnett, S. A., Christensen, K. E., Teat, S.J. \& Allan, D. R. (2012). J. Synchrotron Rad., 19, $435-441$.

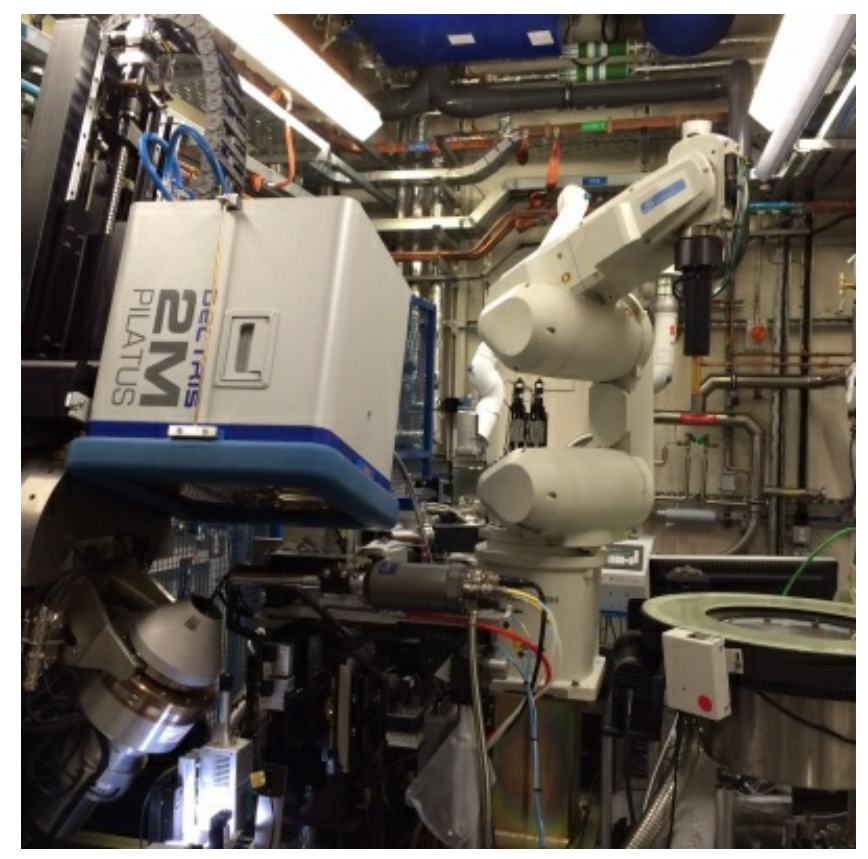

Keywords: synchrotron, remote control, robot 